Review

\title{
Liquid biopsies in lung cancer: The new ambrosia of researchers
}

\author{
Christian Rolfo ${ }^{\mathrm{a}, *, 1}$, Marta Castiglia ${ }^{\mathrm{b}, \mathrm{c}, 1}$, David Hong ${ }^{\mathrm{d}}$, Riccardo Alessandro ${ }^{\mathrm{e}}$, Inge Mertens ${ }^{\mathrm{f}}$, \\ Geert Baggerman ${ }^{\mathrm{f}}$, Karen Zwaenepoel ${ }^{\mathrm{b}}$, Ignacio Gil-Bazo ${ }^{\mathrm{g}}$, Francesco Passiglia ${ }^{\mathrm{a}, \mathrm{c}}$, Anna P. Carreca ${ }^{\mathrm{a}, \mathrm{b}}$, \\ Simona Taverna ${ }^{\mathrm{e}}$, Renza Vento ${ }^{\mathrm{h}}$, Marc Peeters ${ }^{\mathrm{i}}$, Antonio Russo ${ }^{\mathrm{c}}$, Patrick Pauwels ${ }^{\mathrm{b}}$ \\ a Phase I - Early Clinical Trials Unit, Oncology Department, Antwerp University Hospital, Wilrijkstraat 10, Edegem 2650, Belgium \\ b Molecular Pathology Unit, Pathology Department, Antwerp University Hospital, Wilrijkstraat 10, Edegem 2650, Belgium \\ c Department of Surgical, Oncological and Oral Sciences, Section of Medical Oncology, University of Palermo, Via Liborio Giuffrè 5, Palermo 90127, Italy \\ d Department of Investigational Cancer Therapeutics (Phase I Program), The University of Texas MD Anderson Cancer Center, Holcombe Blvd 1400, Unit 455, Houston 77030, USA \\ e Department of Biopathology and Medical and Forensic Biotechnologies, Section of Biology and Genetics, University of Palermo, Via Divisi 81-85, 90133 Palermo, Italy. \\ ${ }^{f}$ Center for Proteomics, VITO, Boeretang 200, Mol, BE-2400, Belgium, Antwerp University, Groenenborgerlaan 171, Antwerpen 2020, Belgium \\ ${ }^{g}$ Lung Cancer Unit, Department of Oncology, Clínica Universidad de Navarra, Avenida Pío XII 36, Pamplona 31008, Spain.

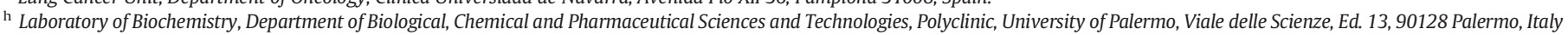 \\ i Oncology Department, Antwerp University Hospital, Wilrijkstraat 10, Edegem 2650, Belgium
}

\section{A R T I C L E I N F O}

\section{Article history:}

Received 23 July 2014

Received in revised form 24 September 2014

Accepted 7 October 2014

Available online 16 October 2014

\section{Keywords:}

Circulating tumor cells

Exosomes

Circulating tumor DNA

Lung cancer

Biomarkers

\begin{abstract}
A B S T R A C T
In the last decades the approach to cancer patient management has been deeply revolutionized. We are moving from a "one-fits-all" strategy to the "personalized medicine" based on the molecular characterization of the tumor. In this new era it is becoming more and more clear that the monitoring of the disease is fundamental for the success of the treatment, thus there is the need of new biomarker discovery. More precisely in the last years the scientific community has started to use the term "liquid biopsy". A liquid biopsy is a liquid biomarker that can be easily isolated from many body fluids (blood, saliva, urine, ascites, pleural effusion, etc.) and, as well as a tissue biopsy, a representative of the tissue from which it is spread. In this review we will focus our attention on circulating tumor cells, circulating tumor DNA, exosomes and secretomes with the aim to underlie their usefulness and potential application in a clinical setting for lung cancer patient management.
\end{abstract}

(c) 2014 Elsevier B.V. All rights reserved.

\section{Contents}

1. Liquid biopsies: definition, usefulness and potential applications . . . . . . . . . . . . . . . . . . . . . . . . . . . . . . . . . . . . 539

2. Clinical perspectives and research opportunities from liquid biopsy in lung cancer . . . . . . . . . . . . . . . . . . . . . . . . . . . . . 541

3. CTCs, ctDNA, exosomes and secretomes in NSCLC: what's new? . . . . . . . . . . . . . . . . . . . . . . . . . . . . . . . . . . . . 541

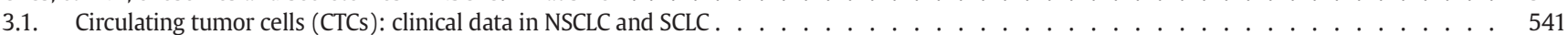

3.2. Circulating tumor DNA (ctDNA): quantitative and qualitative alterations of ctDNA in lung cancer . . . . . . . . . . . . . . . . . . . . 542

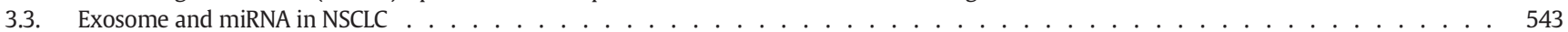

3.4. Secretome in lung cancer . . . . . . . . . . . . . . . . . . . . . . . . . . . . . . . . . . . . . . . . . . . . . . 545

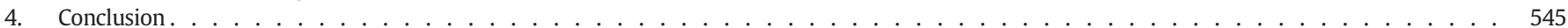

References . . . . . . . . . . . . . . . . . . . . . . . . . . . . . . . . . . . . . . . . . . 545

\footnotetext{
* Corresponding author at: Head of Phase I - Early Clinical Trials Unit, Oncology Department, Antwerp University Hospital, Wilrijkstraat 10, 2650 Edegem, Belgium. Tel.: + 32 38213646; fax: + 3238251592 .

E-mail address: christian.rolfo@uza.be (C. Rolfo).

${ }^{1}$ Both authors contribute equally.
}

\section{Liquid biopsies: definition, usefulness and potential applications}

The term liquid biopsy [1] was originally introduced to define circulating tumor cells (CTCs). Currently it is also used for circulating tumor DNA (ctDNA) [2,3] as well as for exosomes [4] (Fig. 1).

Liquid biopsy analysis is currently a rapidly expanding field in translational cancer research as it might be useful at different points of the diagnostic/therapeutic course of cancer patients. They may be used for 


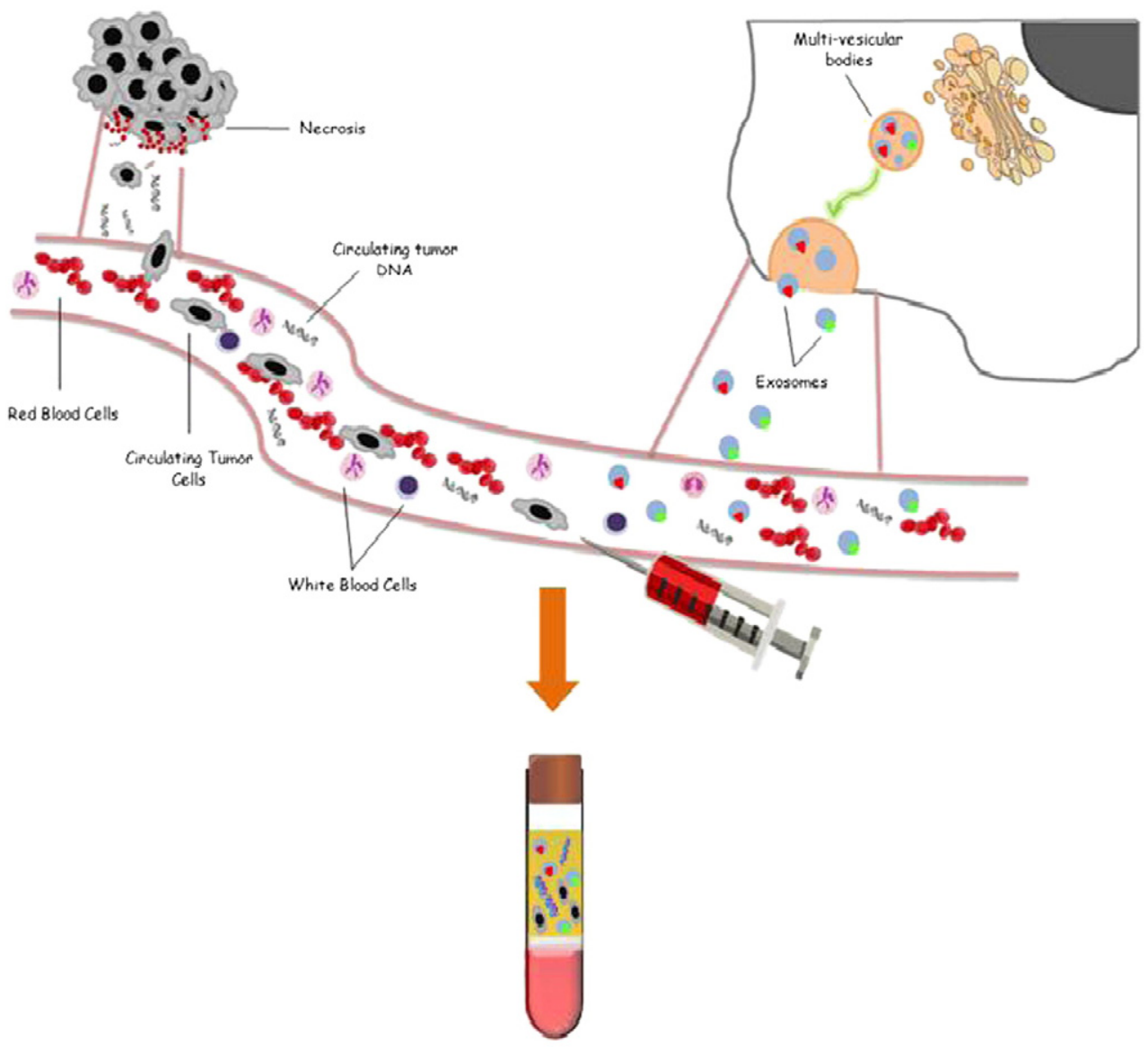

\section{Liquid Biopsy}

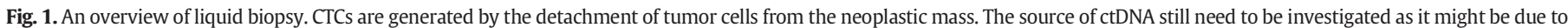

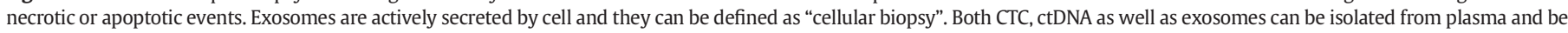
used as a liquid biopsy of the disease.

(a) early diagnosis, (b) estimation of the risk for metastatic relapse or metastatic progression (prognostic information), (c) stratification and real-time monitoring of therapies, (d) identification of therapeutic targets and resistance mechanisms (predictive information), and (e) understanding metastasis development in cancer patients [1]. CTCs, ctDNA and exosomes are likely to contain a wider presentation of genomic data from multiple metastatic sites, whereas mutations present in a single biopsy or minor sub-clone may be missed [5]. Moreover, they may be obtained from almost all body fluids (blood, serum, plasma, urine, pleural effusion, ascites, etc....).

CTCs are formed by cell detachment from the primary tumor mass, determining the migration of tumor cells to secondary sites via the lymphatic and blood system. The presence of CTCs has been demonstrated in the blood of patients with various solid tumors [6] and it has been associated with poor outcome in metastatic non-small cell lung cancer (NSCLC) patients and small cell lung cancer (SCLC) as well as in other tumors $[7,8]$.

As aforementioned, CTC analysis might be useful for both, understanding the metastatic process and also for monitoring the disease during treatment. In this regard there is a wide interest in the utility of circulating nucleic acids (CNAs) and proteins as biomarkers of cancer. Thus far, the majority of research has focused on circulating miRNAs, mRNA and DNA (defined also as cell-free DNA, cfDNA) due to their stability and easy isolation. Circulating miRNA has been extensively studied as a noninvasive biomarker for human disease. The signature profile of circulating miRNA has been shown to distinguish healthy from diseased individuals [9]. Recently the other non-coding RNA
(ncRNA), the long-non-coding RNA (lncRNA) is being investigated as a novel biomarker in cancer patients [10].

cfDNA is released from both healthy and cancer cells but several studies demonstrate that the tumor-released cfDNA (also identified as circulating tumor DNA, ctDNA) is increased in cancer patients when compared to healthy controls and its concentration further increases with advanced disease [11]. The ctDNA can be used for molecular characterization and its exosome-wide analysis could also complement the current invasive biopsy approaches to identify mutations associated with acquired drug resistance in advanced cancers [3] or for treatment outcome monitoring.

Exosomes are small ( 40 to $100 \mathrm{~nm}$ ) membrane derived vesicles that are released extracellularly following the fusion of multivesicular bodies or mature endosomes with the cellular membrane [12,13]. Exosomes are released from normal, diseased, and neoplastic cells and are present in the blood and other body fluids [14]. They contain a variety of molecules such as signal proteins and/or peptides, microRNAs, mRNAs, and lipids [15]. Molecules contained within these small vesicles can therefore move through the bloodstream and reach distant sites, enabling the exchange of "information and materials" between different sites. mRNA-containing exosomes have been shown to be functional, as murine exosomes "taken up" by human cells resulted in the synthesis of mouse proteins [15]. Because exosomes provide signals to distant cells, they act as a nanoparticle-based communication system [16] and could be fundamental for metastasis outcome [17]. Furthermore metastases harbor unique genomic characteristics that may not be detectable in the corresponding primary tumor of the same patients. Thus, the 
mere molecular analysis of the resected primary tumor alone (current standard practice in oncology) or, even the evaluation of tumor characteristics based on the biopsy of the most accessible metastasis may not reveal sufficient information for treatment decisions [18].

Recently, Thakur et al. have demonstrated that exosomes also contain double-stranded DNA, defined as exoDNA, that could be characterized, as well. In the same paper the authors also assessed that exoDNA is representative for the whole genomic DNA and this finding strengthens the translational potential of exosomes as circulating cancer biomarkers [19].

The "secretome" is referred to as the rich, complex set of molecules secreted from living cells. More loosely the term also includes molecules shed from the surface of living cells. Secretome proteins play a key role in cell signaling, communication and migration [20]. The current protein-based serum biomarkers in lung cancer clinical practice include carcinoembryonic antigen (CEA), cytokeratin 19 fragment (CYFRA 211 ), tissue polypeptide antigen (TPA), pro-gastrin releasing peptide (ProGRP), neuron-specific enolase (NSE) and tumor M2 pyruvate kinase [21].

\section{Clinical perspectives and research opportunities from liquid biopsy in lung cancer}

Cancer is not a "molecularly stable" disease, instead it is characterized by a high heterogeneity. In particular for lung cancer it is known that many patients with advanced EGFR-mutated or $A L K$-rearranged tumors do benefit from treatment with specific tyrosine kinase inhibitors (TKIs, such as gefitinib, erlotinib, crizotinib and ceritinib) [22-26]. Unfortunately the length of disease control is notoriously short due to the acquisition of resistance mechanisms. EGFR TKI-acquired resistance is mostly (50-60\%) due to a point mutation in exon 20 (T790M) and occasionally (5-10\%) due to c-MET amplification, among others [27,28]. Thus primary tumor and metastases might be molecularly different. Furthermore, metastasis is the leading cause of death in cancer patients while early-stage disease is starting to be considered curable, or, at least, is characterized by a remarkably high survival rate. A detailed characterization of metastases is therefore needed to improve patient outcome even in advanced-disease stages. The main issue in characterizing metastasis is its intrinsic nature, since it is often tricky and difficult for sampling. Liquid biopsies offer the chance to investigate primary tumor and metastasis through a non-invasive and simple blood tests. Another interesting feature of liquid biopsy, mostly of ctDNA analysis, is the opportunity to study "tumor dormancy" phenomenon. Cell-free DNA might offer the opportunity to monitor patients with no clinically detectable disease after surgery and standard therapy. It has been demonstrated that T790M mutations are detectable in the blood before radiological and symptomatic relapse $[3,29,30]$. Thus in the near future disease monitoring might also be integrated with ctDNA testing and CTC molecular characterization (Fig. 2).

\section{CTCs, ctDNA, exosomes and secretomes in NSCLC: what's new?}

\subsection{Circulating tumor cells (CTCS): clinical data in NSCLC and SCLC}

Since they were first described by Thomas Ashworth in 1869 [31], the presence of circulating tumor cells has been suggested to be a characteristic of cancer by various early studies [32]. CTCs are cancer cells released from tumors into the bloodstream. Over the past years CTCs' presence has been associated with worse prognosis in several major cancer types, including breast, prostate, colorectal and lung cancer. Through a proposed process known as the epithelial-mesenchymal transition (EMT), epithelial cells of solid tumors undergo cellular changes that enable them to escape their structural confines via increased mobility and invasiveness, to enter into the bloodstream, and to adhere to endothelial cells and give birth to distant metastases [33,34].

Thus, isolation and characterization of CTCs represent a very attractive challenge, as they may comprise both the phenotypic and genetic compositions of the primary tumors and potentially serve as a liquid biopsy for any metastatic or non-metastatic tumors. The analysis of CTCs offers the possibility to surrogately evaluate metastasis in real-time and to investigate the actual "molecular condition" of the disease. For example, an EGFR activating mutation can be identified in CTCs and interestingly CTCs offer the opportunity to monitor the appearance of drug resistance mutations (as T790M for TKI resistance) $[35,36]$.

The enumeration of CTCs is considered relevant as a surrogate marker for tumor growth as well as for defining tumor aggressiveness. In this regard, the decrease in the number of CTCs during the treatment has been associated with radiographic tumor response; on the contrary an

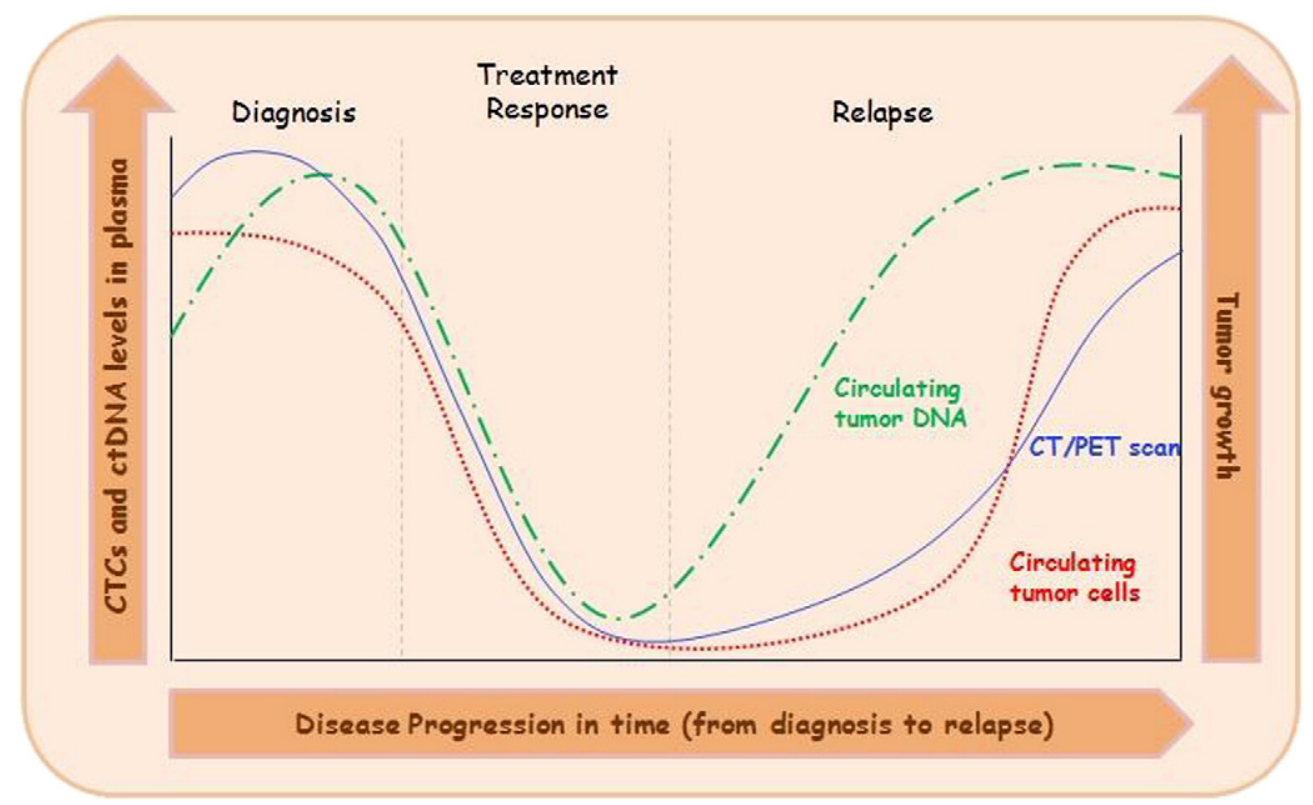

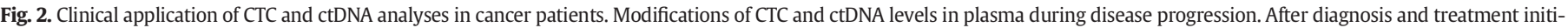

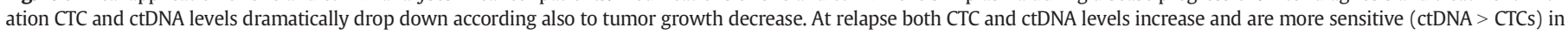
relapse detection than $\mathrm{CT} / \mathrm{PET}$ scan analysis. 
increase in the number of cells predicts tumor progression. Furthermore, in NSCLC patients the total count of CTCs before chemotherapy initiation is associated with staging (higher detectable number of cells in stage IV patients) and the progression-free survival (PFS) and overall survival (OS) seem to be correlated to CTC count. In the study from Krebs G. et al., PFS and OS were higher among NSCLC patients with fewer than five CTCs per $7.5 \mathrm{ml}$ of blood when compared to patients with five or more CTCs [7] and they conclude that CTC number is the strongest predictor of OS. In addition, the presence of circulating cells is associated with a significantly higher risk of lymph node metastasis but not with tumor histology in NSCLC [37]. Recently it has been proved the prognostic value of CTC enumeration in patients with SCLC $[8,38]$. The reduction of CTC count higher than $89 \%$ after one chemotherapy cycle improves prognostic accuracy and is associated with lower risk of death. Furthermore CTC count is strongly associated with the number of metastatic sites [39]. In Table 4 are listed the major clinical investigations of liquid biopsy in lung cancer.

CTCs might be prognostic markers in cancer patients and, in the future, therapy decision should also be made taking into account CTC analysis, especially for the selection of those patients at a higher risk of relapse who might benefit from adjuvant therapies.

Currently, there is only one technology approved for CTC enrichment and enumeration. This technique has been used in various clinical trials (breast, prostate and colon cancers) to establish in which subset of patient CTC count above a known threshold might be used as a prognostic marker and a predictor of patient outcome [40-42]. Based on these clinical trials, the US Food and Drug Administration (FDA) licensed the CellSearch ${ }^{\circledR}$ technology (Veridex, LLC, Raritan, NJ, USA) for CTC analysis in clinical practice. This system uses immunomagnetic purification with antibodies against epithelial cell adhesion molecule (EPCAM) from peripheral blood. The specificity of CellSearch ${ }^{\circledR}$ isolation is only based on EpCAM expression on CTC surface. Consequently, CTCs with low or absent expression of EpCAM are easily missed. Thus, tumor cells that have undergone EMT, and which are probably the most aggressive, are not detectable with CellSearch ${ }^{\circledR}$ technology. There is an increasing number of papers that, by using alternative detection technologies on the basis of EpCAM or other antigens, have demonstrated an apparent greater sensitivity than CellSearch ${ }^{\circledR}[43,44]$ but further studies in the context of larger clinical studies are warranted. In Table 1 are shown the most promising and interesting new techniques for CTC isolation.

While all these new methods of separation provide the advantage of enriching for viable CTCs, which may be collected and stained for cytopathological analysis or subjected to RNA or DNA analyses, the technologies still need to be improved because they are quite elementary and imprecise (especially density gradient and filtration methods). Thus the main issue is to strike a balance between lower number of CTCs but high purity and greater number of CTCs but reduced purity.
Table 2

ctDNA analysis.

\begin{tabular}{llll}
\hline & Methods & Sensitivity & Specificity \\
\hline $\begin{array}{l}\text { Quantitative } \\
\text { analysis }\end{array}$ & *qPCR & High & Low \\
& Amplification of ALU sequences, & & \\
Qualitative analysis & LINE gene, $\beta$-globin, $\beta$-actin & & \\
(based on DNA & Sanger sequencing & Very low & High \\
specific mutation) & Pyrosequencing & Low & Low \\
& *ARMS & Low & High \\
& *BEAMing & Low & High \\
& Digital PCR & High & High \\
& *TAM-Seq & High & High \\
& High & High \\
\hline
\end{tabular}

* qPCR: quantitative polymerase chain reaction (PCR); NGS: Next Generation Sequencing; ARMS: amplification refractory mutation system; BEAMing: beads, emulsion, amplification and magnetic; TAM-Seq: tagged-amplicon deep sequencing.

\subsection{Circulating tumor DNA (ctDNA): quantitative and qualitative alterations of ctDNA in lung cancer}

Currently the evaluation of specific predictive biomarkers is mandatory for a proper treatment of NSCLC patients according to the molecular characterization of the disease. Indeed, the EGFR mutation status assessment in NSCLC patients is definitely embedded in clinical practice. Nonetheless, molecular profiling can be difficult due to the very small amount of tumor tissue available for the analysis (as most are obtained by fine needle aspiration procedures). Many investigators have tried to solve this problem, leading to the development of more sensitive techniques to detect EGFR mutations $[45,46]$. ctDNA testing might be an ideal solution.

ctDNA originates from both healthy and tumor cells $[47,48]$ and may have broad clinical applications because it is non-invasive, convenient and ctDNA based assays can be performed repeatedly [49]. DNA released from necrotic malignant cells varies in size, whereas DNA released from apoptotic cells is uniformly truncated into 185- to 200-bp fragments. Because the main source of free circulating DNA in healthy individuals is from apoptotic cells, a preponderance of longer DNA fragments could be a marker for malignant tumor detection [50,51]. Cellfree DNA levels have been demonstrated to be higher in cancer patients compared with healthy controls [11,52-54]

ctDNA can also be used for mutational analysis and there are already several proof-of-concept papers on the potential of ctDNA testing in the management of NSCLC patients. The whole exome sequencing of ctDNA through Next Generation Sequencing (NGS) has been demonstrated to provide relevant information about the molecular status of the tumor. Recently Murtaza et al. have performed whole exome sequencing of plasma DNA of six patients with advanced cancers (two with breast cancer, three with ovarian cancer and one with NSCLC). Interestingly in NSCLC patients, the analysis of the EGFR gene in CfDNA has shown

Table 1

CTC enrichment and isolation methods: A comparison of the available techniques.

\begin{tabular}{|c|c|c|c|c|c|c|c|c|c|c|c|}
\hline \multirow[t]{2}{*}{ Enrichment methods } & \multirow[t]{2}{*}{ Product } & \multicolumn{2}{|c|}{$\begin{array}{l}\text { Cell } \\
\text { viability }\end{array}$} & \multicolumn{2}{|c|}{$\begin{array}{l}\text { Antibody- } \\
\text { based }\end{array}$} & \multicolumn{2}{|c|}{ Specificity } & \multicolumn{2}{|c|}{ Sensitivity } & \multirow[t]{2}{*}{ Downstream apllication } & \multirow[t]{2}{*}{ References } \\
\hline & & Yes & No & Yes & No & Low & High & Low & High & & \\
\hline \multicolumn{12}{|l|}{ Biological properties } \\
\hline \multirow[t]{4}{*}{ Immunomagnetic bead } & CellSearch System & & $\mathrm{x}$ & $\mathrm{x}$ & & & $\mathrm{x}$ & $\mathrm{x}$ & & CTCs enumeration and analysis & [1] \\
\hline & Magsweeper & $\mathrm{x}$ & & $\mathrm{x}$ & & & $\mathrm{x}$ & $\mathrm{x}$ & & Molecular analysis & [2] \\
\hline & CTC-chip & & $\mathrm{x}$ & $\mathrm{x}$ & & & $\mathrm{x}$ & & $\mathrm{x}$ & CTCs enumeration and analysis & [3] \\
\hline & GILUPI Nanodetector & & $\mathrm{x}$ & $\mathrm{x}$ & & & $\mathrm{x}$ & $\mathrm{x}$ & & CTCs enumeration and analysis & {$[4]$} \\
\hline \multicolumn{12}{|l|}{ Physical properties } \\
\hline Density gradient centrifugation & Immunorosettes & $\mathrm{x}$ & & & $\mathrm{x}$ & $\mathrm{x}$ & & $\mathrm{x}$ & & CTCs enumeration and molecular analysis & {$[5]$} \\
\hline \multirow[t]{2}{*}{ Filtration } & ISET & $\mathrm{x}$ & & & $\mathrm{x}$ & $\mathrm{x}$ & & & $\mathrm{x}$ & CTCs isolation and molecular analysis & [6] \\
\hline & CellSieve & $\mathrm{x}$ & & & $\mathrm{x}$ & $\mathrm{x}$ & & & $\mathrm{x}$ & CTCs isolation and molecular analysis & \\
\hline \multirow{2}{*}{ Dielectrophoresis } & APOstream & $\mathrm{x}$ & & & $\mathrm{x}$ & $\mathrm{x}$ & & & $\mathrm{x}$ & CTCs isolation and molecular analysis & [7] \\
\hline & DEPArray & $\mathrm{x}$ & & & $\mathrm{x}$ & $\mathrm{x}$ & & & $\mathrm{x}$ & CTCs isolation and molecular analysis & [8] \\
\hline
\end{tabular}


Table 3

Exosomes isolation methods.

\begin{tabular}{|c|c|c|c|c|}
\hline Isolation methods & Description & Advantages & Disadvantages & References \\
\hline $\begin{array}{l}\text { Differential } \\
\text { centrifugation }\end{array}$ & 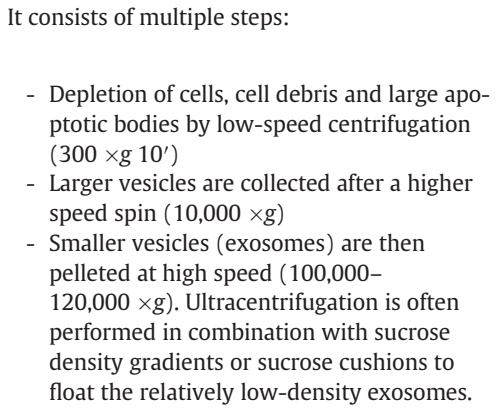 & $\begin{array}{l}\text { - Is considered the gold standard } \\
\text { method } \\
\text { - Is widely used to isolate vesicles } \\
\text { from body fluids and conditioned } \\
\text { medium }\end{array}$ & $\begin{array}{l}\text { - This procedure cannot achieve absolute } \\
\text { separation by size because sedimenta- } \\
\text { tion also depends on the density and } \\
\text { content of vesicles. } \\
\text { - The pellet could contain proteins, com- } \\
\text { plexes, lipoproteins and other contami- } \\
\text { nants. } \\
\text { - Extremely cumbersome process ( } 4-5 \mathrm{~h}) \text {. }\end{array}$ & [9-11] \\
\hline Size exclusion & $\begin{array}{l}\text { Cell fragments and larger vesicles can be } \\
\text { excluded by a filtration step with a } 0.8 \mu \text { m pore } \\
\text { size filter. To collect smaller vesicles } \\
\text { (exosomes), a } 0.2 \mu \mathrm{m} \text { pore size filter can be } \\
\text { used. }\end{array}$ & $\begin{array}{l}\text { - Allow the separation of vesicles } \\
\text { based on size } \\
\text { - Isolation of a vesicle population } \\
\text { homogeneous in size }\end{array}$ & $\begin{array}{l}\text { - Forcing vesicles through a filter could } \\
\text { cause their deformation or breakup. } \\
\text { - Vesicles could be trapped in micro- } \\
\text { pores. }\end{array}$ & [9] \\
\hline $\begin{array}{l}\text { Immunoaffinity } \\
\text { isolation }\end{array}$ & $\begin{array}{l}\text { The presence of characteristic surface proteins } \\
\text { on different vesicle populations, allows the } \\
\text { specific isolation of desired vesicles through the } \\
\text { immunoaffinity isolation, in which antibodies to } \\
\text { surface proteins (CD9, CD63, CD81, MHC) are } \\
\text { used to select desired vesicles population. }\end{array}$ & $\begin{array}{l}\text { High specificity and characterization of } \\
\text { vesicles }\end{array}$ & $\begin{array}{l}\text { - Lower yields because some markers are } \\
\text { not recognized on all vesicles. } \\
\text { - The obtained population cannot be used } \\
\text { for further functional assays. } \\
\text { - It is not suited for isolation of large } \\
\text { amount of vesicles. }\end{array}$ & {$[12-14]$} \\
\hline Microfluidic devices & $\begin{array}{l}\text { Micro- and nano-fluidic methods separate } \\
\text { nanoparticles based on physical properties. }\end{array}$ & $\begin{array}{l}\text { - Reduce material costs and samples } \\
\text { volume } \\
\text { - Single step capture }\end{array}$ & $\begin{array}{l}\text { The application of these methods to } \\
\text { biological fluids has not yet been well } \\
\text { described. }\end{array}$ & {$[15,16]$} \\
\hline $\begin{array}{l}\text { Polymeric } \\
\text { precipitation } \\
\text { (ExoQuick) }\end{array}$ & $\begin{array}{l}\text { Volume-excluding polymers, such us PEGs, are } \\
\text { capable of differentially precipitating vesicles } \\
\text { thereby allowing exosome isolation by low- } \\
\text { speed centrifugation. }\end{array}$ & $\begin{array}{l}\text { - Technically easy } \\
\text { - Require small amount of sample } \\
\text { - Short time of isolation } \\
\text { - high yield of RNA }\end{array}$ & $\begin{array}{l}\text { - The purity of the isolated material is not } \\
\text { certain. } \\
\text { - High contamination from lipoproteins. }\end{array}$ & {$[17,18]$} \\
\hline
\end{tabular}

the occurrence of the resistance mutation T790M at progression, but not at the time of treatment initiation [3]. cfDNA quantification may also be associated to tumor burden estimated by Positron Emission Tomography/Computerized Tomography (PET/CT) [55]. The evaluation of the KRAS mutation in cfDNA from NSCLC individuals has demonstrated that patients with a detectable plasma-KRAS mutation had a significantly shorter OS and PFS compared to patients showing wild type (WT) plasma-KRAS. Thus, the detection of KRAS mutations in plasma may earn a prognostic as well as a predictive value [56].

Despite the promising results obtained in support of the feasibility of ctDNA testing there is still a lack of widely accepted and approved methodologies for ctDNA analysis. To successfully detect the presence of ctDNA in plasma or serum, a suitable methodology needs to be selected. This consists of several essential steps: 1) processing collected blood while avoiding the rupture of blood cell membranes and subsequent plasma contamination with DNA derived from the blood cells nuclei; 2 ) select the most appropriate method to gain a sufficient amount of quality DNA for further analysis; 3) detection of ctDNA (through realtime PCR or digital PCR and NGS) [57]. cfDNA detection can be performed through two main methodologies: quantitative analysis and analysis based on DNA-specific mutation detection. The first one allows DNA quantification of cfDNA including tumor and non-tumor derived DNA. This technique is based on the amplification of ALU sequences or other specific markers ( $\beta$-globin; $\beta$-actin; hTERT; etc....) and detection through real-time PCR [58-61]. DNA-specific mutation analysis in cfDNA is challenging due to the presence of high levels of wild-type DNA originating either from apoptotic or necrotic tissues. Nevertheless mutational studies can be performed using several methods, beginning from sequencing with limited sensitivity, through methods based on conformational changes and electrophoresis (e.g., denaturing capillary electrophoresis, DCE), alternatively increasing the mutant fraction by mutant-enriched PCR (ME-PCR), and opting for a dedicated approach such as "BEAMing" (beads, emulsion, amplification, and magnetics), digital PCR, or single-molecule sequencing [57]. In Table 2 there is an overview of the main techniques used for ctDNA analysis and their characteristics in terms of sensitivity and specificity.

\subsection{Exosome and miRNA in NSCLC}

Exosomes are small membrane vesicles of endocytic origin and were initially isolated from the peripheral circulation of patients with cancer in 1979 [62,63]. Exosomes appear to play a central role in cell-to-cell communication by a direct activation of surface-expressed ligands or by transferring molecules between cells. Exosomes can have a bimodal role in cancer: they can either manipulate the local and systemic environment allowing cancer growth and dissemination or modulate the immune system to elicit or suppress an anti-tumor response $[64,65]$. Exosomes are rich in DNA fragments, proteins, mRNA and microRNA (miRNA). Interestingly enough, exosomal RNA has been shown to determine horizontal transfer of genetic information between cells [66]. In recent years, studies on exosome's prognostic and predictive value in NSCLC have evolved and new evidences have emphasized their potentiality in clinical practice. Biological molecules (protein, RNA and miRNA) well protected by a lipid bilayer membrane that confers high degree of stability, are contained in exosomes and can be isolated and analyzed quite simply. The genome-wide expression profiling of miRNAs has been shown to be significantly different among primary lung cancers and corresponding noncancerous lung tissues and thus has shown to have a potential role as a diagnostic marker. An overexpression of 12 specific miRNAs (hsa-miR-17-3p, hsa-miR-21, hsa-miR106a, hsa-miR-146, hsa-miR-155, hsa-miR-191, hsa-miR-192, hsamiR-203, hsa-miR-205, hsa-miR-210, hsa-miR-212, and hsa-miR-214) has been revealed comparing the miRNA expression profile of lung cancer samples with normal lung tissues [67]. On the basis of this genomewide expression profile, Rabinowits et al. [68] investigated the role of exosomal miRNA as a diagnostic marker for lung cancer. Comparisons between peripheral circulation miRNA-derived exosomes and miRNAderived tumors indicated that the miRNA signatures were not 
significantly different. This approach confirmed that the 12 specific miRNAs were elevated in NSCLC and that the associations of these 12 were mirrored in the circulating exosomes [68]. Thus, from these results, it is suggested that exosome miRNA profiling can be performed in the absence of tumor tissue and accurately reflect the tumor's profile. Exosomal tumor miRNAs have been shown to also educate selected host tissues toward a prometastatic phenotype [69]. This peculiar exosome feature makes them an attractive and interesting target to potentially inhibit the metastatization process.

For translational and clinical practice applications of exosome analysis several features are required: (1) rapidity, exosome-specific extraction, (2) simple, effective biomarker release from exosomes, and (3) highly sensitive, specific detection of endogenous bio-molecules [70]. Conventional exosome diagnostic methods comprise a two-step procedure, including exosome extraction from body fluids, followed by the specific detection of harbored biomarkers that are associated with a variety of diseases [71]. Despite a rapidly growing number of research studies, there is still rather limited and superficial information available regarding RNA content of exosomes [72]. Moreover, certain reports contain a number of contradictions and this is due to the lack of standardized techniques, protocols, and workflows for isolation of exosomes and downstream analysis of their constituents. Currently, the most commonly used approach for exosomes isolation is based on ultracentrifugation [73]. However, this technique is burdened by several problems: the long processing time, the fact that the process is unreliable, unreproducible and not completely selective for tumor exosomes. Furthermore, the abundance of exosomes of different cellular origins and different compositions in peripheral blood makes a further enrichment of the relevant biomarkers a necessary step when considering exosomes for diagnostic or companion diagnostic purposes. In Table 3 the different techniques used to isolate exosomes for prognostic and diagnostic use are summarized.

A viable alternative in distinguishing the different subpopulations of extracellular vesicles (apoptotic bodies, microvesicles and exosomes) might be RNA profiling. In fact, according to RNA profiling, ribosomal RNA (rRNA) is primarily detectable in apoptotic bodies and smaller RNAs without prominent rRNA peaks in exosomes. In contrast, microvesicles contain little or no RNA. The different vesicle pellets show highly different distributions of size, shape and electron density when analyzed by transmission electron microscopy, each having typical characteristics [74]. Currently, the characterization and concentration of nanoparticles in liquid can be obtained through the NanoSight ${ }^{\mathrm{TM}}$ platform [75]. Also other techniques are available for exosome identification and characterization: electron microscopy, flow cytometry and Western blot [76].

Recently the increased application of proteomic technologies has significantly contributed to a deeper understanding of the protein profiles of exosomes from a wide variety of cultured cells and body fluids (such as plasma, urine and malignant effusions) [77]. A part of these studies is specifically focused on tumor derived exosomes (TDE) and the obtained data are providing new knowledge not only for understanding more about their role in the regulation of tumor microenvironment but also for their potential use as disease markers. All proteomics data acquired to date demonstrate that TDEs express a discrete set of proteins specifically related to the tumor phenotype and involved in cell proliferation, antigen presentation, signal transduction, migration, invasion and angiogenesis, supporting the notion that exosomes may play a crucial role in regulating the changes of tumor niche and in driving tumor progression. Several of these proteins can be used for prognostic and diagnostic purposes. Recently, the first global proteomic analysis of highly purified exosomes derived from human NSCLC malignant pleural effusion has been reported. Using nanoLCMS/MS following 1D SDS-PAGE separation, researchers have identified pathologically relevant proteins and potential diagnostic makers for NSCLC, including lung-enriched surface antigens and proteins related to EGFR signaling. These findings provide new insight into the multiple 
functions of exosomes in cancer progression and will aid in the development of novel diagnostic tools for NSCLC [78].

\subsection{Secretome in lung cancer}

The term "secretome" was introduced by Tjalsma et al. in a study of the secreted proteins in Bacillus subtilis [79] and includes proteins released by a cell, tissue or organism through different secretion mechanisms [80]. The cancer secretome was first mentioned in 2006 [81]. Secreted proteins participate in various physiological processes such as immune defense, blood coagulation and cell signaling and also play crucial roles in pathological processes including cancer angiogenesis, differentiation, invasion and metastasis. Proximal biological fluids (e.g. ascites fluid of ovarian cancer or pleural effusion of lung cancer) that are in contact with the tumor may be enriched with proteins secreted or shed by cancer cells. Such proteins could enter the circulation and be detected in body fluids such as blood and urine [20]. The malignant pleural effusion is a clinical complication that occurs relatively often in NSLC. This malignant effusion has been analyzed to identify useful biomarkers in lung cancer and recently a "secretomicprofile" of malignant pleural effusion from lung adenocarcinoma patients has been proposed. Researchers were able to identify 482 nonredundant proteins. Through subsequent in vitro analyses, 4 potential protein biomarkers significantly associated with malignancy were isolated. These findings collectively suggest that the malignant pleural effusion proteome (secretome) from adenocarcinoma NSCLC provides a useful data set for malignancy biomarker research [82]. Several groups have studied the proteomic profile of secreted proteins derived from NSCLC cell lines $[83,84]$ and have tried to translate the obtained results into clinical practice. Despite the fact that the interest in secretome analysis of NSCLC patients is progressively growing, there is still a gap between in vitro secretome testing and clinical applications.

\section{Conclusion}

Our understanding and knowledge of cancer have changed dramatically over the last 30 years. The discovery of oncogene addiction has paved the way for the development of "intelligent" drugs, able to target only cancer cells. The introduction of molecular biomarkers in clinical practice has changed the natural history of many tumors ( $c$-KIT and PDGFRA in GIST, EGFR in lung cancer, BRAF in melanoma and KRAS in colorectal cancer) and the molecular characterization of cancer through NGS still holds many surprises. Nevertheless, the major limitation of these drugs is the rise of resistance mechanisms that impair and slow down the development of genotype-directed therapy in solid tumors.

Tumor cells never reach a steady state. They are continuously changing and they acquire new mutations to survive in an adverse environment. The "dark side" of a targeted agent is due to the selective pressure that favors the selection of most aggressive cells. These cells are likely the ones that escape from the primary tumors and colonize distant districts causing metastasis onset. Since these findings, metastatic cells are thought to be a clone of the primary tumors with a more aggressive behavior. It is now becoming increasingly clear that metastasis and tumors are extremely heterogeneous and therefore, metastatic disease has been proposed as a different condition. From these considerations it is clear how important is the characterization of the new lesion through a re-biopsy. Unfortunately rebiopsy is not always feasible due to many reasons (e.g. comorbidity of the patients, the lack of a safe access to the lesion). In this context, liquid biopsies could be an effective solution. Recently, Oxnard et al. [30] have demonstrated that cell-free plasma DNA is suitable for monitoring treatment response but also for the early detection of acquired resistance mechanisms (e.g. T790M in NSCLC). These and other studies are setting the basis for the development of cfDNA as a new and non-invasive biomarker that can be used routinely as predictive biomarker for targeted therapies.
The value of CTC analysis is, at present, mainly correlated to prognosis but there is an increasing interest in the development of new techniques for their molecular characterization. CTCs are cells that detach from the primary tumors and migrate through the bloodstream. It is not fully understood whether CTCs are the only cause of metastasis but it is well known from clinical studies that their count is proportional to a poorer prognosis in various tumor types. The prognostic value of CTCs is then almost widely accepted while there is still the need of effort for their molecular characterization.

Exosome analysis in cancer is a new field and there are still many questions about their function. Exosomes can be considered as "operating/active secretions" of cancer cells and, as secretome, they might be representative for the functional status of tumor cells. So, these microvesicles are more than simple exophytic budding of cell membrane. Thus, the analysis of molecules contained in exosomes can provide additional information about the tumor biology.

These new insights are paving the way for a deep changing in the approach of patients' management. Analysis based on tissue biopsies will be complemented with the information obtained from CTCs, ctDNA and exosome characterization. These information, together with the one obtained from the imaging analysis, will probably guide treatment decision and will improve the outcome for lung cancer patients. Another important aspect of liquid biopsy is that, due to their non-invasive nature, they might be applied also in a diagnostic setting. It is well known that lung cancer is an insidious disease that most frequently occurs in advanced stage. This is mainly due to the lack of a screening test for the disease and liquid biopsy can be a useful tool for diagnostic purpose.

Nevertheless there are still several limitations for the introduction of liquid biopsy in a clinical setting. Except for CTC enumeration with the Veridex system (FDA-approved), there are no validated techniques both for cfDNA and exosome analysis. cfDNA evaluation is not yet standardized and, for instance, the optimal sampling specimen (plasma or serum) is not yet known. Moreover among laboratories that work with ctDNA there are not yet optimized, standardized and widely approved procedures for blood collection and downstream analysis.

Following this consideration, the increasing effort in liquid biopsy development is understandable, even if the road is still long and winding, but hopes are high to see them included in clinical practice.

\section{References}

[1] C. Alix-Panabieres, K. Pantel, Circulating tumor cells: liquid biopsy of cancer, Clin. Chem. 59 (1) (2013) 110-118.

[2] S.J. Dawson, et al., Analysis of circulating tumor DNA to monitor metastatic breast cancer, N. Engl. J. Med. 368 (13) (2013) 1199-1209.

[3] M. Murtaza, et al., Non-invasive analysis of acquired resistance to cancer therapy by sequencing of plasma DNA, Nature 497 (7447) (2013) 108-112.

[4] J.L. Welton, et al., Proteomics analysis of bladder cancer exosomes, Mol. Cell. Proteomics 9 (6) (2010) 1324-1338.

[5] K.C. Chan, et al., Cancer genome scanning in plasma: detection of tumor-associated copy number aberrations, single-nucleotide variants, and tumoral heterogeneity by massively parallel sequencing, Clin. Chem. 59 (1) (2013) 211-224.

[6] W.J. Allard, et al., Tumor cells circulate in the peripheral blood of all major carcinomas but not in healthy subjects or patients with nonmalignant diseases, Clin. Cancer Res. 10 (20) (2004) 6897-6904.

[7] M.G. Krebs, et al., Evaluation and prognostic significance of circulating tumor cells in patients with non-small-cell lung cancer, J. Clin. Oncol. 29 (12) (2011) 1556-1563.

[8] T.J. Hiltermann, et al., Circulating tumor cells in small-cell lung cancer: a predictive and prognostic factor, Ann. Oncol. 23 (11) (2012) 2937-2942.

[9] X. Chen, et al., Characterization of microRNAs in serum: a novel class of biomarkers for diagnosis of cancer and other diseases, Cell Res. 18 (10) (2008) 997-1006.

[10] D.G. Weber, et al., Evaluation of long noncoding RNA MALAT1 as a candidate bloodbased biomarker for the diagnosis of non-small cell lung cancer, BMC Res. Notes 6 (2013) 518.

[11] S.A. Leon, et al., Free DNA in the serum of cancer patients and the effect of therapy, Cancer Res. 37 (3) (1977) 646-650.

[12] J. Wolfers, et al., Tumor-derived exosomes are a source of shared tumor rejection antigens for CTL cross-priming, Nat. Med. 7 (3) (2001) 297-303.

[13] C. Thery, L. Zitvogel, S. Amigorena, Exosomes: composition, biogenesis and function, Nat. Rev. Immunol. 2 (8) (2002) 569-579.

[14] M. Rodriguez, et al., Different exosome cargo from plasma/bronchoalveolar lavage in non-small-cell lung cancer, Genes Chromosom. Cancer 53 (9) (2014) 713-724. 
[15] H. Valadi, et al., Exosome-mediated transfer of mRNAs and microRNAs is a novel mechanism of genetic exchange between cells, Nat. Cell Biol. 9 (6) (2007) 654-659.

[16] L. Blanc, et al., Exosome release by reticulocytes-an integral part of the red blood cell differentiation system, Blood Cells Mol. Dis. 35 (1) (2005) 21-26.

[17] C. Corrado, et al., Exosomes as intercellular signaling organelles involved in health and disease: basic science and clinical applications, Int. J. Mol. Sci. 14 (3) (2013) 5338-5366.

[18] K. Pantel, C. Alix-Panabieres, Real-time liquid biopsy in cancer patients: fact or fiction? Cancer Res. 73 (21) (2013) 6384-6388.

[19] B.K. Thakur, et al., Double-stranded DNA in exosomes: a novel biomarker in cancer detection, Cell Res. 24 (6) (2014) 766-769.

[20] M.P. Pavlou, E.P. Diamandis, The cancer cell secretome: a good source for discovering biomarkers? J. Proteome 73 (10) (2010) 1896-1906.

[21] R.V. Polisetty, et al., Glioblastoma cell secretome: analysis of three glioblastoma cell lines reveal 148 non-redundant proteins, J. Proteome 74 (10) (2011) 1918-1925.

[22] D. Torti, L. Trusolino, Oncogene addiction as a foundational rationale for targeted anti-cancer therapy: promises and perils, EMBO Mol. Med. 3 (11) (2011) 623-636.

[23] G. Bronte, et al., Driver mutations and differential sensitivity to targeted therapies: a new approach to the treatment of lung adenocarcinoma, Cancer Treat. Rev. 36 (Suppl. 3) (2010) S21-S29.

[24] M. Soda, et al., Identification of the transforming EML4-ALK fusion gene in nonsmall-cell lung cancer, Nature 448 (7153) (2007) 561-566.

[25] C. Rolfo, et al., Looking for a new panacea in ALK-rearranged NSCLC: may be Ceritinib? Expert Opin. Ther. Targets 18 (9) (2014) 983-985.

[26] G. Bronte, et al., Are erlotinib and gefitinib interchangeable, opposite or complementary for non-small cell lung cancer treatment? Biological, pharmacological and clinical aspects, Crit. Rev. Oncol. Hematol. 89 (2) (2014) 300-313.

[27] C.E. Steuer, S.S. Ramalingam, ALK-positive non-small cell lung cancer: mechanisms of resistance and emerging treatment options, Cancer 120 (16) (2014) 2392-2402.

[28] C. Rolfo, et al., Novel therapeutic strategies for patients with NSCLC that do not respond to treatment with EGFR inhibitors, Cancer Treat. Rev. 40 (8) (2014) 990-1004.

[29] K. Taniguchi, et al., Quantitative detection of EGFR mutations in circulating tumor DNA derived from lung adenocarcinomas, Clin. Cancer Res. 17 (24) (2011) $7808-7815$.

[30] G.R. Oxnard, et al., Noninvasive detection of response and resistance in EGFRmutant lung cancer using quantitative next-generation genotyping of cell-free plasma DNA, Clin. Cancer Res. 20 (6) (2014) 1698-1705.

[31] T. Ashworth, A case of cancer in which cells similar to those in the tumours were seen in the blood after death, Med. J. Aust. 14 (1869) 146-147.

[32] M.V. Gallivan, J.J. Lokich, Carcinocythemia (carcinoma cell leukemia). Report of two cases with English literature review, Cancer 53 (5) (1984) 1100-1102.

[33] J.P. Thiery, Epithelial-mesenchymal transitions in tumour progression, Nat. Rev. Cancer 2 (6) (2002) 442-454.

[34] P.S. Steeg, Metastasis suppressors alter the signal transduction of cancer cells, Nat. Rev. Cancer 3 (1) (2003) 55-63.

[35] S. Maheswaran, et al., Detection of mutations in EGFR in circulating lung-cancer cells, N. Engl. J. Med. 359 (4) (2008) 366-377.

[36] D.B. Costa, Identification of somatic genomic alterations in circulating tumors cells: another step forward in non-small-cell lung cancer? J. Clin. Oncol. 31 (18) (2013) 2236-2239.

[37] J. Huang, et al., Prognostic significance of circulating tumor cells in non-small-cell lung cancer patients: a meta-analysis, PLoS One 8 (11) (2013) e78070.

[38] S. Igawa, et al., Circulating tumor cells as a prognostic factor in patients with small cell lung cancer, Oncol. Lett. 7 (5) (2014) 1469-1473.

[39] N. Normanno, et al., Prognostic value of circulating tumor cells' reduction in patients with extensive small-cell lung cancer, Lung Cancer 85 (2) (2014) 314-319.

[40] D.F. Hayes, et al., Circulating tumor cells at each follow-up time point during therapy of metastatic breast cancer patients predict progression-free and overall survival, Clin. Cancer Res. 12 (14 Pt 1) (2006) 4218-4224.

[41] D.C. Danila, et al., Circulating tumor cell number and prognosis in progressive castration-resistant prostate cancer, Clin. Cancer Res. 13 (23) (2007) 7053-7058.

[42] S.J. Cohen, et al., Relationship of circulating tumor cells to tumor response, progression-free survival, and overall survival in patients with metastatic colorectal cancer, J. Clin. Oncol. 26 (19) (2008) 3213-3221.

[43] C. Wu, et al., Preliminary investigation of the clinical significance of detecting circulating tumor cells enriched from lung cancer patients, J. Thorac. Oncol. 4 (1) (2009) 30-36.

[44] S. Nagrath, et al., Isolation of rare circulating tumour cells in cancer patients by microchip technology, Nature 450 (7173) (2007) 1235-1239.

[45] T. John, G. Liu, M.S. Tsao, Overview of molecular testing in non-small-cell lung cancer: mutational analysis, gene copy number, protein expression and other biomarkers of EGFR for the prediction of response to tyrosine kinase inhibitors, Oncogene 28 (Suppl. 1) (2009) S14-S23.

[46] A. Marchetti, et al., EGFR mutations in non-small-cell lung cancer: analysis of a large series of cases and development of a rapid and sensitive method for diagnostic screening with potential implications on pharmacologic treatment, J. Clin. Oncol. 23 (4) (2005) 857-865

[47] M. Stroun, et al., The origin and mechanism of circulating DNA, Ann. N. Y. Acad. Sci. 906 (2000) 161-168.

[48] K.L. Aung, et al., Current status and future potential of somatic mutation testing from circulating free DNA in patients with solid tumours, Hugo J. 4 (1-4) (2010) 11-21.

[49] M. Utting, et al., Microsatellite analysis of free tumor DNA in urine, serum, and plasma of patients: a minimally invasive method for the detection of bladder cancer, Clin. Cancer Res. 8 (1) (2002) 35-40.
[50] S. Jahr, et al., DNA fragments in the blood plasma of cancer patients: quantitations and evidence for their origin from apoptotic and necrotic cells, Cancer Res. 61 (4) (2001) 1659-1665.

[51] M.B. Giacona, et al., Cell-free DNA in human blood plasma: length measurements in patients with pancreatic cancer and healthy controls, Pancreas 17 (1) (1998) 89-97.

[52] X.Y. Zhong, et al., Elevated level of cell-free plasma DNA is associated with breast cancer, Arch. Gynecol. Obstet. 276 (4) (2007) 327-331.

[53] G. Sozzi, et al., Quantification of free circulating DNA as a diagnostic marker in lung cancer, J. Clin. Oncol. 21 (21) (2003) 3902-3908.

[54] X. Chen, et al., Detecting tumor-related alterations in plasma or serum DNA of patients diagnosed with breast cancer, Clin. Cancer Res. 5 (9) (1999) 2297-2303.

[55] A.D. Nygaard, et al., The correlation between cell-free DNA and tumour burden was estimated by PET/CT in patients with advanced NSCLC, Br. J. Cancer 110 (2) (2014) 363-368.

[56] A.D. Nygaard, et al., The prognostic value of KRAS mutated plasma DNA in advanced non-small cell lung cancer, Lung Cancer 79 (3) (2013) 312-317.

[57] L. Benesova, et al., Mutation-based detection and monitoring of cell-free tumor DNA in peripheral blood of cancer patients, Anal. Biochem. 433 (2) (2013) 227-234.

[58] M. van der Vaart, P.J. Pretorius, Is the role of circulating DNA as a biomarker of cancer being prematurely overrated? Clin. Biochem. 43 (1-2) (2010) 26-36.

[59] N. Umetani, et al., Increased integrity of free circulating DNA in sera of patients with colorectal or periampullary cancer: direct quantitative PCR for ALU repeats, Clin. Chem. 52 (6) (2006) 1062-1069.

[60] K.B. Sriram, et al., Pleural fluid cell-free DNA integrity index to identify cytologically negative malignant pleural effusions including mesotheliomas, BMC Cancer 12 (2012) 428.

[61] M. Zane, et al., Circulating cell-free DNA, SLC5A8 and SLC26A4 hypermethylation, BRAF(V600E): a non-invasive tool panel for early detection of thyroid cancer, Biomed. Pharmacother. 67 (8) (2013) 723-730.

[62] D.D. Taylor, G.J. Doellgast, Quantitation of peroxidase-antibody binding to membrane fragments using column chromatography, Anal. Biochem. 98 (1) (1979) 53-59.

[63] D.D. Taylor, H.D. Homesley, G.J. Doellgast, Binding of specific peroxidase-labeled antibody to placental-type phosphatase on tumor-derived membrane fragments, Cancer Res. 40 (11) (1980) 4064-4069.

[64] H.G. Zhang, W.E. Grizzle, Exosomes and cancer: a newly described pathway of immune suppression, Clin. Cancer Res. 17 (5) (2011) 959-964.

[65] C. Kahlert, R. Kalluri, Exosomes in tumor microenvironment influence cancer progression and metastasis, J. Mol. Med. (Berl.) 91 (4) (2013) 431-437.

[66] J. Ratajczak, et al., Embryonic stem cell-derived microvesicles reprogram hematopoietic progenitors: evidence for horizontal transfer of mRNA and protein delivery, Leukemia 20 (5) (2006) 847-856.

[67] N. Yanaihara, et al., Unique microRNA molecular profiles in lung cancer diagnosis and prognosis, Cancer Cell 9 (3) (2006) 189-198.

[68] G. Rabinowits, et al., Exosomal microRNA: a diagnostic marker for lung cancer, Clin. Lung Cancer 10 (1) (2009) 42-46.

[69] S. Rana, K. Malinowska, M. Zoller, Exosomal tumor microRNA modulates premetastatic organ cells, Neoplasia 15 (3) (2013) 281-295.

[70] F. Wei, J. Yang, D.T. Wong, Detection of exosomal biomarker by electric field-induced release and measurement (EFIRM), Biosens. Bioelectron. 44 (2013) 115-121.

[71] C. Chen, et al., Microfluidic isolation and transcriptome analysis of serum microvesicles, Lab Chip 10 (4) (2010) 505-511.

[72] S. Mathivanan, et al., ExoCarta 2012: database of exosomal proteins, RNA and lipids, Nucleic Acids Res. 40 (Database issue) (2012) D1241-D1244.

[73] C. Thery, et al., Isolation and characterization of exosomes from cell culture supernatants and biological fluids, Curr. Protoc. Cell Biol. (2006) Chapter 3: p. Unit 322. Suppl. 30: p. 3.22.1-3.22.29.

[74] R. Crescitelli, et al., Distinct RNA profiles in subpopulations of extracellular vesicles: apoptotic bodies, microvesicles and exosomes, J. Extracell. Vesicles 2 (2013).

[75] J. Webber, A. Clayton, How pure are your vesicles? J. Extracell. Vesicles 2 (2013).

[76] C. Lasser, M. Eldh, J. Lotvall, Isolation and characterization of RNA-containing exosomes, J. Vis. Exp. (59) (2012) e3037.

[77] S. Fontana, et al., Contribution of proteomics to understanding the role of tumorderived exosomes in cancer progression: state of the art and new perspectives, Proteomics 13 (10-11) (2013) 1581-1594.

[78] J.O. Park, et al., Identification and characterization of proteins isolated from microvesicles derived from human lung cancer pleural effusions, Proteomics 13 (14) (2013) 2125-2134.

[79] H. Tjalsma, et al., Signal peptide-dependent protein transport in Bacillus subtilis: a genome-based survey of the secretome, Microbiol. Mol. Biol. Rev. 64 (3) (2000) 515-547.

[80] M.W. Volmer, et al., Differential proteome analysis of conditioned media to detect Smad4 regulated secreted biomarkers in colon cancer, Proteomics 5 (10) (2005) 2587-2601.

[81] M. Gronborg, et al., Biomarker discovery from pancreatic cancer secretome using a differential proteomic approach, Mol. Cell. Proteomics 5 (1) (2006) 157-171.

[82] C.J. Yu, et al., Comprehensive proteome analysis of malignant pleural effusion for lung cancer biomarker discovery by using multidimensional protein identification technology, J. Proteome Res. 10 (10) (2011) 4671-4682.

[83] T. Xiao, et al., An approach to studying lung cancer-related proteins in human blood, Mol. Cell. Proteomics 4 (10) (2005) 1480-1486

[84] Y. Chen, et al., Elevation of serum l-lactate dehydrogenase B correlated with the clinical stage of lung cancer, Lung Cancer 54 (1) (2006) 95-102. 